\title{
Estimating Radiation Therapy Toxicity and Tolerability with Comprehensive Assessment Parameters in Geriatric Cancer Patients
}

\author{
Sukran Ulger ${ }^{1 *}$, Muhammet Cemal Kizilarslanoglu², Mustafa Kemal Kilic², \\ Diclehan Kilic ${ }^{1}$, Bekir Eren Cetin ${ }^{1}$, Zekeriya Ulger ${ }^{2}$, Eray Karahacioglu ${ }^{1}$
}

\begin{abstract}
Cancer prevalance and incidence is increasing with aging of populations and age is a critical factor in decision-making for anti-cancer treatment. However it is believed that chronological age is not enough to guide management in elderly cancer patients. Multidisciplinary evaluation and comprehensive geriatric assessment has gained importance regarding the treatment selection especially for definitive anti-cancer therapy recently. We here aimed to analyse the effect of the comprehensive geriatric assessment parameters on radiotherapy toxicity and tolerability in a series of geriatric cancer patients in Turkey.
\end{abstract}

Keywords: Comprehensive assessment - elderly - cancer - radiotherapy - geriatric oncology

Asian Pac J Cancer Prev, 16 (5), 1965-1969

\section{Introduction}

Cancer prevalence and incidence increases with age and the mortality rates are higher in patients over 65 years of age (Pal and Hurria, 2010; Rodrigues and Sanatani, 2012). One of the major risk factors for both the development of cancer and poor tolerability of cancer treatment is patients' chronological age (Rodrigues and Sanatani, 2012). However, basing a treatment plan primarily on a patient's chronological age will lead to inadequate therapy for geriatric cancer patients, especially those who could tolerate treatment and have a chance of survival with an aggressive and curative approach (Varol et al., 2014).

Clinical trials on the elderly cancer population are limited due to the increased mortality rates associated with patients' comorbidities and the fact that advanced age itself can exacerbate the management and outcome complexity (Yancik et al., 1999). There is limited data for this group of patients regarding treatment selection, tolerability, and outcomes related to age (Rodrigues and Sanatani, 2012). Moreover clinicians are more prone to treat elderly patients with conservative approaches (Inamoto et al., 2014; Oran et al., 2014). However, it is recommended that elderly cancer patients be discussed by multidisciplinary boards, including geriatricians, in order to optimize patient care, enhance the effectiveness of teatment, reduce treatment toxicity, and increase survival rates (Aliamus et al., 2011; Rodrigues and Sanatani, 2012).
Before their physicians make a decision regarding anticancer treatment in the elderly, it is suggested that patients undergo a comprehensive geriatric assessment (CGA) that includes an evaluation of the patient's clinical, physical, psychological, social, and mental status (Gomez-Millan, 2009; Le Fur E et al., 2013). The possible benefits of CGAs include the ability to predict complications and side effects of treatment, estimate survival rates, improve pain control, and assisting the oncological treatment decision-making. However, there is very limited data about this issue studies (Freyer et al., 2005; Rao et al., 2005; Ramjaun et al., 2013; Hamaker et al., 2014). Although guidelines recommend a CGA before treatment decision-making for elderly cancer patients, there has been little documentation regarding how to determine CGA tools. Moreover there is no widespread usage in routine oncological practice (Extermann et al., 2005). The data Show the impact of CGA tools on the outcome of cancer treatment in geriatric patients is lacking. The data on the effects of a CGA on the outcome of radiation therapy (radiation toxicity, local control, and survival) in geriatric individuals is also very limited (Spyropoulou et al., 2014). Furthermore, to the best of our knowledge, there has never been a study that shows the relationship between CGAs and cancer treatment tolerability in geriatric cancer patients receiving curative radiation therapy (RT).

In this study, we aim to analyze the relationship between CGA results and the toxicity and tolerability of curative radiation therapy in geriatric cancer patients. 


\section{Materials and Methods}

\section{Study population}

Thirty geriatric cancer patients who have been admitted to our radiation oncology department are prospectively included in this study. All patients have a diagnosis of cancer managed by curative radiation therapy. Before initiation of the RT, the patients were referred to the Geriatrics Department for evaluation of their status and CGA. After completion of the geriatric examination, all patients underwent curative conventional RT for their primary tumor. The patients were evaluated at 3 months post-completion of RT and all acute toxicities related to the treatment were recorded. The study was approved by the Local Ethical Committee and all members of the study population signed the informed consent form.

\section{Comprehensive geriatric assessment tool}

All patients were evaluated by geriatricians. The following parameters were used in the CGA: activities of daily living (ADL) (Mahoney and Barthel, 1965), instrumental activities of daily living (IADL) (Lawton Table 1. Patients Characteristics

\begin{tabular}{lcc}
\hline & No. of Patients & $\%$ \\
\hline Mean age, years & $70 \pm 6$ & \\
Gender (Male) & 22 & 73.3 \\
Smoking cigarette & 10 & 33.3 \\
Alcohol usage & 2 & 6.7 \\
Types of cancer & & \\
Prostate & 8 & 26.7 \\
Lung & 5 & 16.7 \\
Gastrointestinal tumor & 4 & 13.3 \\
Breast & 3 & 10 \\
Brain tumor & 3 & 10 \\
Larynx & 2 & 6.7 \\
Gynecological tumor & 2 & 6.7 \\
Liposarcoma & 2 & 6.7 \\
Diffuse large B cell lymphoma & 1 & 3.3 \\
Hypertension & 14 & 46.7 \\
Coronary artery disease & 1 & 3.3 \\
Diabetes Mellitus & 5 & 16.7 \\
Depression & 2 & 6.7 \\
Sarcopenia & 3 & 10 \\
Asthma & 1 & 3.3 \\
\hline
\end{tabular}

Table 2. Toxicity Related to Radiotherapy

\begin{tabular}{llcc}
\hline & & No of Patients & $\%$ \\
\hline Cystitis & & 8 & 26.7 \\
& GrI & 6 & \\
Dermatitis & GrII & 2 & 20 \\
& GrI & 6 & \\
& GrII & 4 & 20 \\
& Esophagitis & 6 & \\
& GrI & 4 & 13.3 \\
Proctitis & GrII & 2 & \\
& GrI & 4 & 10 \\
Emesis & GrII & 3 & \\
& GrI & 1 & 66.7 \\
& Total & 3 & \\
& & 27 toxicities & \\
& & (in 20 patients) & \\
\hline
\end{tabular}

and Brody,1969) mini-mental state examination (MMSE) (Folstein et al.,1975), clock drawing test (CDT) (ShuaHaim et al., 1996), mini nutritional assessment (MNA) (Rubenstein et al., 2001), geriatric depression scale (GDS) handgrip strength (HS) (Yesavage et al., 1982), 6 meters gait speed, up and go test (Mathias et al., 1986), calf circumference and serum vitamin D levels.

Patients' functional dependency was evaluated by using ADL and IADL tests. Cognitive functions were evaluated using MMSE and CDT tests. An MNA-short form test was used to evaluate the patients' nutritional status. The depression status of the participants was evaluated according to the geriatric depression scale. Detailed information about all the tests used for evaluation is available online and may be located using cited references.

To evaluate the patients' physical performance and to identify the patient's level of sarcopenia, the HS test, 6 meters gait speed test, and calf circumference measurements were used. Handgrip strength was measured by using a standard grip strength dynamometer (Grip D, produced by Takei, made in Japan). Three different measurements (in kilograms) were taken from the patients' dominant hand and the highest measurement was accepted as the test score. Calf-circumference was measured in centimeters by using a standard tape measure. The Up and Go test was used to estimate the patients' risk of falling.

\section{Evaluation of radiotherapy toxicities}

The patients were evaluated in terms of acute toxicities during and after three months of post-completion of radiotherapy by using Radiation Therapy Oncology Group (RTOG) scoring system (Cox et al., 1995) (The grading system used for all tissues and systems was 1,2,3 and 4).

\section{Statistical analyses}

All the variables were entered into the SPSS (Statistical Package for Social Sciences) program version 15.0 for statistical analyses. Kolmogorov-Smirnov and ShapiroWilk tests were used to determine which parameters had normal distribution. Numerical parameters normally distributed were shown as mean \pm SD and skew distributed ones were shown as median (min-max). Statistical differences between numerical parameters in groups were tested by using a student t-test for normally distributed variables and the Mann-Whitney $U$ test for skew distributed parameters. Pearson's $\mathrm{x} 2$ test was used for the evaluation of differences between categorical variables. A p-value $<0.05$ was considered statistically significant.

\section{Results}

A total of 30 elderly cancer patients undergoing curative RT were prospectively followed and analyzed in this study. All patients underwent a geriatric examination and CGA. The physical examination and CGA parameters were recorded by a geriatrician. Radiation therapy plannings were applied as three-dimensional conformal radiation therapy (3DCRT) or intensity modulated radiation therapy (IMRT). The type of RT administered 

Table 3. Comparison of CGA Parameters Results between Patients with and without Radiotherapy Toxicities

\begin{tabular}{lccc}
\hline Parameters & Patients with toxicity & Patients without toxicity & P value \\
\hline MMSE score $($ mean \pm SD) & $28.4 \pm 1.18$ & $28.1 \pm 3.28$ & 0.785 \\
CDT score (mean \pm SD) & $5.7 \pm 0.7$ & $5.6 \pm 1.0$ & 0.572 \\
MNA-SF score (mean \pm SD) & $11.3 \pm 1.6$ & $11.5 \pm 2.2$ & 0.774 \\
GDS score (median) & 1 & 0.5 & 0.559 \\
ADL score (median) & 0 & 0 & 1.000 \\
IADL score (mean \pm SD) & $23.8 \pm 0.8$ & $22.8 \pm 1.9$ & 0.165 \\
Gait speed (m/s) (mean \pm SD) & $1.3 \pm 0.4$ & $1.3 \pm 0.3$ & 0.797 \\
Up and go test score (median) & 1 & 1 & 0.846 \\
HS $(\mathrm{kg}$ ) (mean \pm SD) & $33.8 \pm 9.3$ & $27.6 \pm 7.6$ & 0.077 \\
CC $(\mathrm{cm})($ mean \pm SD) & $34.1 \pm 3.6$ & $35.6 \pm 5.1$ & 0.382 \\
Vitamin D level $(\mathrm{ng} / \mathrm{ml})$ & $15.0 \pm 10.0$ & $24.2 \pm 13.1$ & 0.164 \\
\hline
\end{tabular}

*MMSE: Mini-mental state examination, CDT: Clock drawing test, MNA-SF: Mini-nutritional assessment short form, GDS: Geriatric depression scale, ADL: Activities of daily living, IADL: Instrumental activities of daily living, HS: Hand-grip strength, CC: Calf-circumference

was determined by the primary tumor site and dose volume histogram parameters for each patient. The prescribed radiation dose was min $45 \mathrm{~Gy}$, max 74 Gy with a daily 1, 8-2 Gy Daily fractionation. All patients completed their prescribed radiation doses and were followed by the radiation oncologist on a weekly basis in order to record acute toxicities. Two patients with lung tumors, 1 patient with a laryngeal tumor, and 1 patient with gastric cancer received concomitant chemotherapy (CT). Thirteen patients $(43.3 \%)$ received CT before radiotherapy. The toxicities were evaluated according to the RTOG acute toxicity scoring system and recorded.

The mean age of the patients was $70 \pm 6$ years and $73.3 \%$ of the patients were male. The general characteristics of the patients are shown in Table 1 . The most common cancer types in the study population were prostate $(26.7 \%)$, lung $(16.7 \%)$, and breast cancer $(10.0 \%)$.

During and after the completion of radiotherapy, $20(66.7 \%)$ patients experienced radiotherapy-related toxicities. A total of 27 toxicities manifested as follows: 8 cystitis (26.7\%), 6 dermatitis (20\%), 6 esophagitis (20\%), 4 proctitis (13.3\%), and 3 emesis (10\%) (details are shown in Table 2). According to the RTOG scoring system, all toxicities related to the radiotherapy were either grade 1 or grade 2 . There were no grade 3 or 4 toxicities as a result of the treatment nor any treatment-related deaths on follow-up.

The statistical analysis used to evaluate the relationship between the CGA parameters and the presence or absence of RT toxicities determined that there were no differences between groups regarding CGA parameters (results are shown in Table 3). However, when the CGA parameters were analyzed for each toxicity, the results indicated that the patients with esophagitis had significantly lower vitamin D levels compared to those without esophagitis (5.3 ng/ml vs $21.3 \mathrm{ng} / \mathrm{ml}, \mathrm{p}=0.022)$. The second statistically significant correlation was shown between the patients with emesis and the patients with decreased gait speed $(0.88 \mathrm{~m} / \mathrm{s}$ vs $1.3 \mathrm{~m} / \mathrm{s}, \mathrm{p}=0.039)$. There was no statistically significant correlation between cystitis, dermatitis, and proctitis and CGA parameters.

\section{Discussion}

The main conclusion of the current study is that a
CGA is important when making a decision regarding definitive radiation therapy in elderly patients. The results of our study showed that two parameters of CGA, vitamin D deficiency and decreased gait speed (an important parameter of frailty), correlated to the radiotherapy toxicities in geriatric cancer patients and that these parameters can be used as simple tools in routine clinical practice to estimate the tolerability of cancer treatment in the elderly population. We did not find any significant relationship between the other CGA parameters and radiation toxicities. We believe this was due to the general good performance status of our study population, all of whom tolerated the CT and/or RT.

There is a growing concern about CGAs is available in geriatric oncology and it is emphasized that geriatric patients should be evaluated by a geriatrician before initiation of treatment in order to anticipate the results of treatment (Extermann et al., 2005; Extermann et al., 2007; Brugel et al., 2013). In order to determine the impact of CGAs on the prognosis of the geriatric cancer population, some studies have been conducted. The results of these limited studies have shown that a CGA is more useful and effective than the evaluation of patients according to performance status or age (Kanesvaran et al., 2011; Puts et al., 2014). However, thus far there have not been any studies that focus on the ability of CGA parameters to predict radiotherapy toxicities and tolerability. In general, the published studies have focused on the relationship between the toxicities and tolerability of CT and CGA parameters in the elderly (Puts et al., 2014). As the data about CGA parameters and cancer RT in geriatric patients is lacking, a study showing the relationship between definitive RT toxicities and tolerability and CGA parameters in elderly cancer patients was emergent. This study is organized as a preliminary study because it is the first study in this area. We are planning to do well designed studies about this topic. Follow-up visits will be conducted with the current study's patient population in order to evaluate the relationship between the treatment outcome and CGA parameters.

One of the major findings of the present study is that geriatric cancer patients with vitamin D deficiency are more prone to the development of esophagitis compared to a group of patients without vitamin D deficiency. This result has shown the importance of vitamin D deficiency 
in relation to treatment tolerability and it also supports the finding that vitamin $\mathrm{D}$ deficiency may cause poor prognosis in cancer (Li et al., 2014). Besides the wellknown association between vitamin D deficiency and an increased risk for different cancer types and poor prognosis in cancer, many experimental studies have shown the effect of vitamin D on the stimulation of innate immunity and suppression of acquired immunity (Arnson et al., 2007; Ma et al., 2011). Regarding its ability to modulate the patient's immune response it is thought that vitamin D may play an important role in the development of esophagitis (which is an inflammatory response to radiation). Observational studies have shown that calcitriol reduces the proliferation of some cytokines with inflammatory properties and stimulates the production of other cytokines with anti-inflammatory actions (Bruyere et al., 2014). The results of the present study suggest that vitamin $\mathrm{D}$ is also a key factor in the inflammatory response to radiation.

Another important aspect of our study is the significant association between decreased gait speed and emesis. It is well-known that decreased gait speed is one of the primary criteria of frailty in the elderly population. The results of our study showed that patients who have a decreased gait speed are more susceptible to the development of emesis while receiving radiotherapy than are patients with a normal gait speed. We hypothesize that this result may be explained by problems with the central nervous system or inner ear. Decreased gait speed is a result of gait and balance problems mainly related to the patient's balance systems in the central nervous system or balance organs such as the inner ear. However, detailed descriptions of this problem are not available in the current literature and the knowledge is limited. Radiotherapy-induced emesis is not completely documented and explained in the current literature either. It is generally believed that this complication develops as a result of the same mechanisms at work in chemotherapy-induced emesis. The major causative structures which provoke the development of emesis are related to neuroanatomy and neurotransmitters (Borison and Wang, 1953; Naylor and Inall, 1994). Our study results are the first to indicate a significant correlation between decreased gait speed and emesis. This is a new and exciting area that warrants investigation with larger studies.

The current study has some limitations. First, the number of patients included in the study was limited. Primary tumors and RT doses. were not similar in consequence of owing the participants different types of cancer. The general performance status of all patients was well and they all had acceptable CGA results. Despite these limitations, we believe this study offers valuable preliminary findings in the area of geriatric oncology.

In conclusion, the present study is the first study to examine the relationship between CGA results and curative radiotherapy toxicities in geriatric cancer patients. The results of the study indicate that vitamin D deficiency and decreased gait speed might be useful and simple predictors for estimating RT toxicities and tolerability in geriatric cancer patients. The results of our study have emphasized the significance of CGA parameters in making decisions about how to manage the treatment of geriatric cancer patients. Further studies are needed in order to determine the correlation between specific CGA parameters and treatment tolerability and cancer outcome.

\section{References}

Aliamus V,Adam C, Druet-Cabanac M, Dantoine T, Vergnenegre A (2011). Geriatric assessment contribution to treatment decision-making in thoracic oncology. Revue Des Maladies Respiratoires, 28, 1124-30.

Arnson Y, Amital H, Shoenfeld Y (2007). Vitamin D and autoimmunity: new aetiological and therapeutic considerations. Ann Rheum Dis, 66, 1137-42.

Borison HL, Wang SC (1953). Physiology and pharmacology of vomiting. Pharmacol Rev, 5, 193-230.

Brugel L, Laurent M, Caillet P, et al (2014). Impact of comprehensive geriatric assessment on survival, function, and nutritional status in elderly patients with head and neck cancer: protocol for a multicentre randomised controlled trial (EGeSOR). BMC Cancer, 14, 427.

Bruyere O, Cavalier E, Souberbielle JC, et al (2014). Effects of vitamin D in the elderly population: current status and perspectives. Arch Public Health, 28, 32.

Cox JD, Stetz J, Pajak TF (1995). Toxicity criteria of the Radiation Therapy Oncology Group (RTOG) and the European Organization for Research and Treatment of Cancer (EORTC). Intern J Radiat Oncol Biol Phys, 31, 1341-6.

Durdux C, Bauer C (2008). Radiation therapy in elderly patients. Cancer Radiotherapie, 12, 548-53.

Durdux C, Boisserie T, Gisselbrecht M (2009). Radiation therapy in elderly patients. Cancer Radiotherapie, 13, 609-14.

Extermann M, Aapro M, Bernabei R, et al (2005). Task force on CGA of the international society of geriatric oncology. use of comprehensive geriatric assessment in older cancer patients: recommendations from the task force on CGA of the international society of geriatric oncology (SIOG). Critical Rev Oncol Hematol, 55, 241-52.

Extermann M, Hurria A (2007). Comprehensive geriatric assessment for older patients with cancer. J Clinical Oncol, 25, 1824-31.

Folstein MF, Folstein SE, McHugh PR (1975). "Mini-mental state". A practical method for grading the cognitive state of patients for the clinician. J Psychiatric Res, 12, 189-98.

Freyer G, Geay JF, Touzet S, et al (2005). Comprehensive geriatric assessment predicts tolerance to chemotherapy and survival in elderly patients with advanced ovarian carcinoma: a GINECO study. Ann Oncol, 16, 1795-800.

Gomez-Millan J (2009). Radiation therapy in the elderly: more side effects and complications? Critical Rev Oncol Hematol, 71, 70-8.

Hamaker ME, Prins MC, Stauder R (2014). The relevance of a geriatric assessment for elderly patients with a haematological malignancy-a systematic review. Leukemia Res, 38, 275-83.

Inamoto T, Azuma H, Nonomura N, et al (2014). Favorable outcome in elderly Asian patients with metastatic renal cell carcinoma treated with everolimus: the Osaka urologic oncology group. Asian Pac J Cancer Prev, 15, 1811-5.

Kanesvaran R, Li H, Koo KN, Poon D (2011). Analysis of prognostic factors of comprehensive geriatric assessment and development of a clinical scoring system in elderly Asian patients with cancer. J Clin Oncol, 29, 3620-7.

Lawton MP, Brody EM (1969). Assessment of older people: self-maintaining and instrumental activities of daily living. Gerontologist, 9, 179-86. 
Le Fur E, Chatellier G, Berger A, et al (2013). Tolerance and efficacy of preoperative radiation therapy for elderly patients treated for rectal cancer. Cancer Radiotherapie, 17, 202-7.

Li M, Chen P, Li J, et al (2014). Review: the impacts of circulating 25-hydroxyvitamin D levels on cancer patient outcomes: a systematic review and meta-analysis. J Clin Endocrinol Metabol, 99, 2327-36.

Ma Y, Zhang P, Wang F, et al (2011). Association between vitamin $D$ and risk of colorectal cancer: a systematic review of prospective studies. J Bone Miner Res, 29, 3775-82.

Mahoney FI, Barthel DW (1965). Functional evaluation: the barthel index. Maryland State Med J, 14, 61-5.

Mathias S, Nayak US, Isaacs B (1986). Balance in elderly patients: the "get-up and go" test. Arch Phys Med Rehabilitat, 67, 387-9.

Naylor RJ, Inall FC (1994). The physiology and pharmacology of postoperative nausea and vomiting. Anaesthesia, 49, 2-5.

Oran ES, Yankol Y, Soybir GR, et al (2014). Distinct postsurgical management in young and elderly breast cancer patients results in equal survival rates. Asian Pac J Cancer Prev, 15, 7843-7.

Pal SK, Hurria A (2010). Impact of age, sex, and comorbidity on cancer therapy and disease progression. J Clin Oncol, 28, 4086-93.

Puts MT, Santos B, Hardt J, et al (2014 ). An update on a systematic review of the use of geriatric assessment for older adults in oncology. Ann Oncol, 25, 307-15.

Rao AV, Hsieh F, Feussner JR, Cohen HJ (2005). Geriatric evaluation and management units in the care of the frail elderly cancer patient. J Gerontol Series A, Biological Sci Med Sci, 60, 798-803.

Ramjaun A, Nassif MO, Krotneva S, Huang AR, Meguerditchian AN (2013). Improved targeting of cancer care for older patients: a systematic review of the utility of comprehensive geriatric assessment. J Geriatric Oncol, 4, 271-81.

Rodrigues G, Sanatani M (2012). Age and comorbidity considerations related to radiotherapy and chemotherapy administration. Seminars Radiat Oncol, 22, 277-83.

Rubenstein LZ, Harker JO, Salva A, Guigoz Y, Vellas B (2001). Screening for undernutrition in geriatric practice: developing the short-form mini-nutritional assessment (MNA-SF). $J$ Gerontol Series A, Biological Sci Med Sci, 56, 366-72.

Shua-Haim J, Koppuzha G, Gross J (1996). A simple scoring system for clock drawing in patients with Alzheimer's disease. J Am Geriatrics Soc, 44, 335.

Spyropoulou D, Pallis AG, Leotsinidis M, Kardamakis D (2014). Completion of radiotherapy is associated with the Vulnerable Elders Survey-13 score in elderly patients with cancer. $J$ Geriatric Oncol, 5, 20-5.

Varol U, Dirican A, Yildiz I, et al (2014). First-line monochemotherapy in frail elderly patients with metastatic colorectal cancer. Asian Pac J Cancer Prev, 15, 3157-61.

Yancik R, Yates JW, Cumberlin R (1999). Research recommendations for radiation therapy in older cancer patients. report from the national institute on aging, National cancer institute, and American college of radiology Workshop: radiation therapy and cancer in older persons. Intern J Radiat Oncol, Biol, Phys, 43, 3-5.

Yesavage JA, Brink TL, Rose TL, et al (1982). Development and validation of a geriatric depression screening scale: a preliminary report. J Psychiatric Res, 17, 37-49. 